\title{
TOWARDS A NEW METHOD FOR KINEMATIC QUANTIFICATION OF BRADYKINESIA IN PATIENTS WITH PARKINSON'S DISEASE USING TRIAXIAL ACCELOMETRY
}

\author{
Peter H. Veltink1, Erwin G. Olde Engberink1, Bob J. van Hilten 2 , Rob Dunnewold2 and Cathrien Jacobi2 \\ IUniversity of Twente, P.O. Box 217, 7500 AE Enschede, the Netherlands \\ 2Dept. of Neurology, University Hospital Leiden, Leiden, the Netherlands
}

\begin{abstract}
We propose a new kinematic analysis procedure using triaxial accelerometers mounted to the wrist in the assessment of bradykinesia in patients with Parkinson's disease (PD). The deviation of the magnitude of the accelerometer vector signal from the magnitude of the gravitational acceleration is taken as a measure for effective magnitude of the acceleration at the position of the triaxial accelerometer. For low acceleration, two of the three angles describing the orientation of the lower arm can be derived from the accelerometer signals.
\end{abstract}

\section{Introduction}

Parkinson disease (PD) is clinically characterized by a variable expression of four cardinal features: impaired voluntary movement, resting tremor, rigidity, and impaired postural stability. The reduction of movement (hypokinesia), and the slow excecution of movement (bradykinesia) are the two tiers of the altered voluntary movement which show considerable improvement with levodopa treatment. Timed performance tests are used in the assessment of bradykinesia. The momentary severity of bradykinesia, however, may vary highly in time, which makes any assessment that relies on a spot check unreliable. Consequently, there is a need for an objective continuous assessment procedure which preferably can be performed in the home setting over a longer period of time.

Previously, actometers have been used for such measurements (Van Hilten et al., [1]). Actometers can be considered as binary uniaxial accelerometers, counting the times the acceleration exceeds a certain threshold. Although actometers appeared to give a good indication of total movement activity, bradykinesia can not be quantified.

Several methods have been proposed to use accelerometers for quantification of movement disorders in PD ([2], [3]). Frost [2] used triaxial piezoelectric accelerometer at the wrist, hand, ankle or foot and proposed performance indices derived from the magnitude of the total acceleration vector and its derivative. His method is limited to the analysis of paced and spatially controlled range-ofmotion tasks. Van Someren et al. [3] proposed a method to distinguish tremor from underlying movements using one piezoelectric accelerometer. It was based on an analysis of repetitive periodic movements within a certain frequency range (tremor). Bradykinesia was quantified using the amplitude of the periods classified as underlying movement.

In this paper we propose a new method for quantification bradykinesia, taking account of the gravity and acceleration components in the accelerometer signal, yielding orientation information in near static situations and quantifying acceleration in dynamic situations. For this reason we use accelerometers of the piezoresistive type, which also measure DC components during prolonged static periods.

\section{Theory}

The signal $\vec{s}$ of a triaxial accelerometer can be expressed as the sum of the actual acceleration vector $\bar{a}$ and the gravitational acceleration $\overrightarrow{\boldsymbol{g}}$ :

$$
\overrightarrow{\boldsymbol{s}}=\vec{a}-\overrightarrow{\boldsymbol{g}}
$$

Under dynamic conditions the components $\bar{a}$ and $\bar{g}$ are indistinguishable in the signal $\vec{s}$. However, under near static conditions $(\|\vec{a}\|<<\|\vec{g}\|)$ the signal $\vec{s}$ mainly exists of the gravitational acceleration vector $\vec{g}$. Near static conditions can be detected directly from the accelerometer signal:

1. $\|\vec{s}\| \approx\|\vec{g}\|$

2. the magnitude of $\vec{s}$ does not vary significantly over time and the direction of $\vec{s}$ only varies slowly.

We propose to use the signal $s_{f}$ being the high-pass filtered, rectified and low-pass filtered signal $\|\vec{s}\|-\|\vec{g}\|:$

$$
s_{f}=L P F\{R E C[H P F(\|\vec{s}\|-\|\bar{g}\|)]\}
$$

The following criterion for near static condition can be used:

$$
s_{f} \leq s_{t}
$$

with $s_{t}$ being a preset threshold.

In near static periods two of the three angles describing the orientation of the triaxial accelerometer, and thus of the body segment to.which it is attached, can be tracked continuously. More specifically, the angles $\varphi_{1}$ (elevation out of the horizontal plane) and $\varphi_{2}$ (axial orientation of the segment) can be determined, but not angle $\varphi_{3}$ (orientation around the vertical axis):

$$
\varphi_{1}=\arctan \left(\frac{s_{1}}{\sqrt{s_{2}^{2}+s_{3}^{2}}}\right)
$$




$$
\varphi_{2}=\arctan \left(\frac{s_{3}}{s_{2}}\right)
$$

with $s_{1}$ the radial component (in the axial direction of the body segment) and $s_{2}$ and $s_{3}$ the tangential components of the triaxial accelerometer signal $\vec{s}$. The validity range of the arctan function can be extended to a full 360 degree range by taking account of the signs of the nominator and denominator of the argument.

In dynamic periods the triaxial accelerometer signal does not give accurate information about the orientation of the accelerometer. However the signal $s_{f}$ is a measure for the acceleration $\|\bar{a}\|$, because of the triangular inequality:

$\|\vec{a}\| \leq\|\vec{s}\|-\|\vec{g}\|$

\section{Methods}

The kinematic analysis procedure described above was tested in preliminary experiments in healthy subjects. A triaxial accelerometer, consisting of 3 uniaxial piezoresistive accelerometers (ICsensors, 5g), was attached at the lower arm of the subject near the wrist. The subject was asked to perform cyclical movements of the lower arm with a range of 90 degrees in a vertical plane, with the elbow at a fixed position. The movement started at a low frequency, which was gradually increased, resulting in a fast cyclical movement, and subsequently gradually decreased.

\section{Preliminary Results}

Figure 1 shows some preliminary results of the cyclical movement tests. As expected, the signal $s_{f}$ gives a measure for the effective acceleration magnitude $\|\vec{a}\|$ (fig. 2a). The estimate of angle $\varphi_{1}$ (fig. 2b) appears to be only slightly effected at fast movements. Angle $\varphi_{2}$ (fig. 2c) was fairly constant during the test, but the reconstructions showed deviations around vertical arm position (once every cycle), when $\varphi_{2}$ is not observable from signals $s_{2}$ and $s_{3}$, and small measurement errors can yield erroneous readings.

\section{Discussion}

Our preliminary results show the feasibility of kinematic analysis in the assessment of bradykinesia using the identified orientations of the lower arm in time for near static circumstances and the signal $s_{f}$ for faster movements. The influence of the magnitude of signal $s_{f}$ on the error in the angle estimates remains to be further analyzed. Additionally, the feasibility of this procedure in PD patients with predominant resting tremor needs to be investigated.

Tremor and underlying movement may be seperated by time-frequency analysis (e.g. van Someren et al. [3]). The actual angle calculation by the method presented in this paper provides additional means for this seperation.
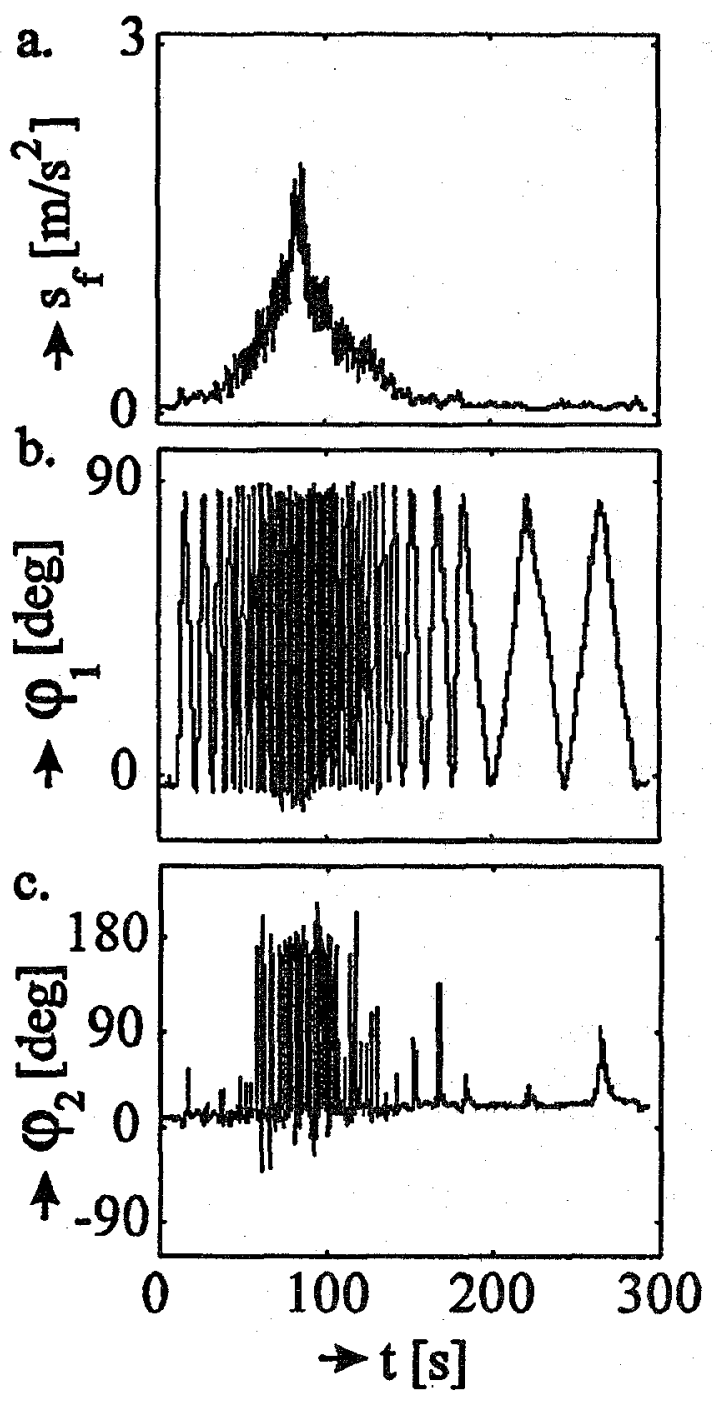

Fig. 1. Preliminary experimental results of cyclical lower arm movement test: $a: s_{f} ; b: \varphi_{1} ; c: \varphi_{2}$.

\section{References}

[1] J.J. van Hilten, G. Hoogland, E.A. van der Velde, G.A. Kerkhof, J.G. van Dijk and R.A.C. Roos, "Quantitative Assessment of Parkinsonian Patients by Continuous wrist activity monitoring", Clin. Neuropharmacol., vol. 16, 1993, pp. 36-45.

[2] J.D. Frost, "Triaxial Accelerometry: A Method for Quantifying Tremor and Ataxia", IEEE Trans. Biomedical Engineering, vol. 25, 1978, pp. 17-27.

[3] E.J.W. van Someren, W.A. van Gool, B.F.M. Vonk, M. Mirmiran, J.D. Speelman, D.A. Bosch and D.F. Swaab, "Ambulatory Monitoring of Tremor and Other Movements before and after Thalomotomy: a new Quantitative Technique", J. of Neurological Sciences, vol. 117, 1993, pp. 16-23. 